Aus der otiatrischen Abteilung des Kaiser Franz-Josef-Ambulatoriums in Wien.

(Vorstand: Dozent Dr. H. Fre y.)

\title{
Beitrag zur Kenntnis der frühzeitigen familiären Otosklerose.
}

\author{
Von \\ Dr. E. Paul-Boncour (Paris).
}

In der Lehre von der Otosklerose lassen sich mehrere Entwicklungsperioden unterscheiden. In der ersten Periode war man sich über die pathologische Definition dieser Erkrankung noch nicht im klaren und, wie bekannt, wurden chronischkatarrhalische Adhäsivprozesse und Residualprozesse nach Entzündungen und Eiterungen mit ihr vielfach zusammengeworfen. Diese erste Periode fand ihren Abschluß durch die ausgedehnten Untersuchungen $\mathrm{P}$ ol i t $\mathrm{z}$ ers, der zum ersten Male an einem größeren Materiale ein streng umschriebenes, anatomisches Bild dieser Erkrankung aufzeigte und es in Beziehung zu einem bestimmten, regelmäßig sich wiederholenden klinischen Symptomenkomplex setzte. Nach ihm handelte es sich bei der typischen Otosklerose um primäre Knochenveränderungen in der Kapsel des Labyrinthes, verbunden mit den eindeutigen klinischen Zeichen einer fortschreitenden Erkrankung des schalleitenden Apparates. Eine Anzahl von Arbeiten anderer Autoren, von denen insbesonders $\mathrm{S}$ i e b e $\mathrm{n}$ mann, $\mathrm{Hab}$ ermann und $\mathrm{Bezold}$ erwähnt werden mögen, befestigte die Ansichten Politzers in bezug auf den anatomischen Tatbestand; nur die Genese des Prozesses wurde verschieden beurteilt, und die zweite Periode in der Geschichte der Otosklerose war ausgefüllt mit Diskussionen über die Frage, ob es sich um eine primäre oder sekundäre, von der Schleimhaut ausgehende Erkrankung des Knochens handelt, bis endlich die Auffassung im Sinne Politzers 
allgemein akzeptiert schien. Neuerdings aber findet man in der Literatur Anzeichen, als ob diese Ansichten in ihrer Gültigkeit wieder beeinträchtigt werden sollten, und zwar teils durch anatomische, teils durch klinische Befunde, die von den betreffenden Autoren für genügend erachtet werden, um die Charakteristik der Otosklerose als einer primären Erkrankung des schalleitenden Apparates zu bezweifeln. Indem ich bezüglich der Literatur über diese Frage auf die ausführlichen

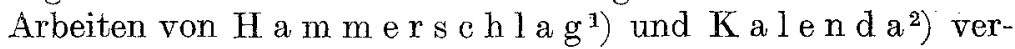
weise, möchte ich mich hier nur mit der klinischen Seite der Frage befassen.

$\mathrm{K}$ a 1 en d a beschreibt den Fall eines 23 jährigen jungen Mannes, der mit 11 Jahren einen Typhus durchgemacht hatte, von dem er allerdings ohne Ohraffektion genas. Der Patient wurde ungefähr 1/4 Jahr nach dem Eintreten der ersten subjektiven Störungen untersucht und zeigte beiderseits den für die Otosklerose als typisch angenommenen rosenroten Schimmer am Trommelfell. Der Funktionsprüfungsbefund zeigte auf dem rechten Ohre eine Kombination von Störungen, entsprechend einem Schalleitungshindernis und eine Affektion des schallperzipierenden Apparates, während auf dem linken Ohre eine ähnliche Kombination mit außerordentlichem Überwiegen der Störungen von seiten des inneren Ohres bestand. K a $\mathrm{l}$ e $\mathrm{n}$ d a schließt nun aus diesem Verhalten, daß sein Fall ein ,weiteres Glied einer bereits recht stattlich angewachsenen Beweiskette sei, welche schließlich vielleicht zu einer Modifikation in der dermaligen Lehre von der Otosklerose führen kann".

Mit dieser Modifikation ist die Anschauung gemeint, daß es sich bei der Otosklerose um primäre Erkrankungen des inneren Ohres handeln kann, bei denen die Erscheinungen im Schalleitungsapparat nur in zweiter Linie in Betracht kämen.

Diese Anschauung, durch welche die Auffassung der Otosklerose als einer abgegrenzten primären Erkrankung des schall l e it end en Apparates mit eventuellen späteren Veränderungen des schall perzipierenden Apparates erschüttert wird, geht parallel mit derjenigen von Autoren, die aus anatomischen Befunden die Meinung gewonnen haben, daß die otosklerotischen Veränderungen sowohl im Bereiche

1) Zeitschrift für Ohrenheilkunde Bd. 59, S. 315, sowie Monatsschrift für Ohrenheilkunde Bd. 44, Heft 7, S. 6, 7, 9.

2) Zeitschrift für Ohrenheilkunde Bd. 60, S. 229. 
des schalleitenden als des schallperzipierenden Apparates beginnen können.

M a n a s s e ${ }^{1}$ glaubt sogar, daß Zweifel berechtigt sind, ob die bekannten anatomischen Knochenveränderungen überhaupt als Ursachen der klinischen Erscheinungen anzusehen seien, und befindet sich dabei in Übereinstimmung mit $\mathrm{H}$ e g e $n$ e $r^{2}$ ), der sagt: ,dagegen weisen eine ganze Reihe von Erwägungen darauf hin, daß man es bei der Otosklerose nicht mit einem sekundären Prozesse im Akustikus, sondern einem primären, parallel und unabhängig von der Knochenerkrankung verlaufenden, manchmal auch schon vor Beginn derselben einsetzenden Frkrankung desselben zu tun hat".

Wir sehen demnach, daß teils vom klinischen, teils vom anatomischen Standpunkte aus Bedenken erhoben werden gegen die Auffassung der Otosklerose im früheren Sinne; von einem anderen Standpunkte aus gelangt $\mathrm{H}$ a $\mathrm{m}$ m e r s c h 1 a g ${ }^{3}$ ) zu ähnlichen Folgerungen. Indem er das ätiologische Moment in Betracht zieht, findet er eine nahe Verwandtschaft zwischen Otosklerose, kongenitaler hereditärer Taubstummheit und gleichwertiger hereditärer labyrinthärer Schwerhörigkeit, die nach ihm ,nur verschiedene Erscheinungsformen einer einzigen, genetisch einheitlichen Erkrankung darstellen'.

Angesichts dieser Unsicherheit, die den bisher festgehaltenen Begriff der Otosklerose bedroht, scheint es mir nun gerechtfertigt, auf eine Beobachtung hinzuweisen, die ich an der otiatrischen Abteilung des Kaiser Franz Josef-Ambulatoriums in Wien machen konnte ${ }^{i}$ ). Eis handelt sich um 2 Knaben im Alter von 12 und 14 Jahren, die Halbbrüder sind Söhne einer Mutter, aber zweier verschiedener Väter. Der klinische Bericht ist der folgende:

I. I. B., $12 \mathrm{~J}$ a h $\mathrm{r}$ e a.lt. Patient litt im Alter von 3 Jahren an einer tuberkulösen Erkrankung des rechten Kniegelenkes, an der er längere Zeit behandelt wurde. Es soll damals auch eine Otorrhoe bestanden haben, die nach kurzer Zeit sistierte, worauf das Gehör wieder vollständig gut gewesen sein soll.

Seit zwei Jahren glaubt man zu Hause und in der Schule eine Abnahme des Gehöres zu bemerken; anderweitige Störungen fielen nicht auf; subjektive Geräusche werden auf Befragen in beiden Ohren angegeben.

1) Verhandlungen der deutschen otologisehen Gesellschaft 1909, 18. Versammlung in Basel 1909, S. 234.

2) ibidem S. 84 .

3) 1. c.

4) Die beiden $z u$ beschreibenden Fälle wurden von meinem Chef, Herrn Dozenten Dr. H. Fr e y in der österreichischen otologischen Gesellschaft, Sitzung vom 31. Oktober 1910, vorgestellt. 
Die Untersuchung ergab zu wiederholten Malen folgenden ubereinstimmenden Befund:

Trommelfell L: normal, mit einem schwachen rosenroten Schimmer in hinteren unteren Quadranten, R: mehr opak, ebenfalls rötlicher Schimmer, aber undeutlicher.

Funktions-Prüfung:

Konvers.-Spr.

Flüster-Spr.

Uhr

e (Luftleitung)

Schwabach

Rinne

Weber

$\mathrm{C}_{1}$

$\mathrm{c}_{4}$

Galton-Edelmann

Uhr in Knochenleitung
$\mathrm{R}$

$41 / 2 \mathrm{~m}$

$11 / 2 \mathrm{~m}$

$15 / 300$

- 41 sek.

- 9 Sek.

- (11 Sek.)

u n bestim t

$-34$

$-3 \frac{1}{2}$

0,4
L

$3 \mathrm{~m}$

$3 / 4 \mathrm{~m}$

$15 / 300$

- 59 Sek.

+ 10 Sek.

- (15 Sek.)

$-45$

$-4$

0,4 (normale Girenze)

Die sorgfältige Prufung des Vestibularapparates ergab keinerlei Zeichen einer Störung.

Luftdusche und Vibrationsmassage bewirken keine Veränderung des Hörvermögens.

II. L. B., Halbbruder des Vorgenannten, $14 \mathrm{~J}$ ah re alt, bemerkt seit ungefähr einem Jahre Abnahme des Gehörs, temporär subjektive Geräusche; öfter Kopfschmerz; frühere Krankheiten werden nicht angegeben.

Trommelfellbefund beiderseits normal mit Ausnahme eines dentlichen rosenroten Schimmers im hinteren unteren Abschnitt bis gegen den Umbo zu, rechts etwas deutlicher als links.

Funktions-Prüfung:

Konvers.-Spr.

Flüster-Spr.

Chr

e in Luftleitung

Schwabach

Rinne

Weber

$\mathrm{C}_{1}$

$\mathrm{c}_{4}$

Galton-Edelmann

Uhr in Knochenleitung.
R

$7 \mathrm{~m}$

$31 / 2 \mathrm{~m}$

$8 / 300$

- 34 Sek.

+13 Sek.

- (18)

rechts

$-35$

unverkürzt

0,4
L

$7 \mathrm{~m}$

$41 / 2 \mathrm{~m}$

$12 / 300$

- 18 Sek.

+ 14 Sek.

- (11)

$-32$

unverkürzt

0,4 (normale Grenze)

Die Reaktionen des Vestibularapparates zeigen normales Verhalten, Therapie wie im vorhergehenden Falle ohne Einfluß.

Es ist zweifellos, daß es sich in diesen beiden Fällen um typische Otosklerose im bisherigen Sinne handelt. Das Bemerkenswerte an ihnen scheint mir nun darin zu liegen:

Beide Fälle stellen sich nach dem Funktionsprüfungsbefund als eindeutige Erkrankungen des Schalleitungsapparates dar. Im Falle II besteht allerdings eine geringe Verkürzung der Hördauer für $c_{4}$, ein Umstand, der aber gegenüber den anderen Erscheinungen sehr in den Hintergrund tritt und gerade bei dem Falle zu finden ist, dessen Krankheitsdauer die des anderen wesentlich übertrifft. Weiterhin kommt in 
Betracht, daß es sich um zwei sehr jugendliche Individuen handelt, bei denen die Otosklerose noch verhältnismäBig selten auftritt, wenn dies aber der Fall ist, in der Regel in intensiver Weise. Nun sind aber ferner diese Fälle sicherlich als familiär disponierte, als hereditäre, wenn man will, degenerative, anzusehen, da sie Blutsverwandte betreffen. Hier sei auch darauf hingewiesen, daß die beiden Individuen mütterlicherseits verwandt sind, da schon öfter darauf aufmerksam gemacht wurde, daß sich die Otosklerose vorzugsweise in der weiblichen Linie vererbt. Wenn es sich nun hier auch um zwei Individuen männlichen Geschlechts handelt, so ist doch ihre familiäre Disposition nur auf die Mutter zurückzuführen, da sie das einzige Bindeglied zwischen beiden Patienten darstellt. Für den Ausgangspunkt dieser hereditären Anlage fehlt uns freilich jeder Anhalt, da die Mutter trotz gründlichen Befragens keine Fälle von Schwerhörigkeit in ihrer Familie zu kennen vorgibt.

Es liegen demnach hier zwei Fälle vor, in denen eine hereditäre Erkrankung des Gehörorganes mit verhältnismäßig schon bedeutender Herabsetzung des Hörvermögens aufgetreten ist, mit dem typischen Trommelfellbefund der Otosklerose und den Erscheinungen eines $\mathrm{Sch}$ alleitung s$\mathrm{h}$ indernisses, die also sämtlichen Anforderungen des klassischen Typus Politzers gerecht werden. Nun kann kein Zweifel bestehen, daß gerade solche Fälle diejenigen sind, an die wir uns zunächst halten müssen, um allgemein gültige Beobachtungen über die Otosklerose anzustellen, da sie wohl nach jeder Richtung hin als einwandfreie angesehen werden können, und wenn es uns demnach gelungen ist, zwei solche typische Fälle familiärer Otosklerose in einem frühen Stadium ihrer Entwicklung zu untersuchen, so können wir aus dem Befunde auch die Berechtigung ableiten, zu sagen, daß die Otosklerose keinesfalls, wie manche der eingangs erwähnten Autoren meinen, als eine primäre Erkrankung des schallperzipierenden Apparates beginnen muß, sondern daß sie in typischer Form als eine Affektion des Schalleitungsapparates auch in jenen Fällen beginnen kann, die man zu den degenerativen im Sinne der Autoren rechnen muß.

Demgegenüber können Fälle wie der von $\mathrm{K}$ a l e n d a doch nur als ausnahmsweise Kombination gelten, und wenn man auch $\mathrm{H}$ a m m e s chlag ohne weiteres beistimmen kann, wenn er Otosklerose und Taubstummheit als hereditäre Degenerativprozesse bezeichnet, so muß man sich dennoch davor hüten, dieselben zu identifizieren. 
Nischfälle beweisen höchstens, daß degenerative Zustände und ibre Folgen gleichzeitig an verschiedenen Stellen eines Organes auftreten können, ebenso wie sie an verschiedenen Organen desselben Individuums oder an verschiedenen Mitgliedern derselben Familie gleich oder verschieden sich zeigen können. Man darf aber darüber nicht vergessen, daß es ein wohlcharakterisiertes Bild der Otosklerose gibt, das sich in zahllosen klinischen Fällen als das eines reinen Schalleitungshindernisses darstellt und für das die eben geschilderten ein Beispiel abgeben, wie man es in einem so frühen Stadium nicht häufig zu beobachten imstande ist.

Ich möchte deshalb zusammenfassend schließen: Solange es Fälle gibt, die sich von allen Gesichtspunkten aus als typische Bilder des klinisch feststehenden Begriffes der Otosklerose darstellen und gleichzeitig in einem sehr frühen Stadium nur reine Störungen des schalleitenden Apparates aufweisen, kann weder die Annahme, daß die Otosklerose gleichzeitig mit Veränderungen des Perzeptions- und des Leitungsapparates beginne, noch die, daß sie eine primär vom Perzeptionsapparat ausgehende Erkrankung sei, in ihrer Allgemeinheit zu Recht bestehen. 